\title{
La salud del hombre en la perspectiva de la sexualidad: un relato de experiencia
}

\author{
Men's health in the sexuality perspective: an \\ experience report
}

\section{A saúde do homem na perspectiva da sexualidade: um relato de experiência}

\author{
Janaína Paula Calheiros Pereira Sobral ${ }^{1}$, Alana Maiara Brito \\ Bibiano $^{2}$, Marília Martina Guanaany de Oliveira Tenórioº ${ }^{3}$ Taíse \\ Gama dos Santos ${ }^{4}$, Roberto Firpo de Almeida Filho ${ }^{5}$, Nívia Madja \\ dos Santos Silva ${ }^{6}$
}

\begin{abstract}
${ }^{1}$ Enfermeira, Mestranda em Enfermagem, Universidade Federal de Alagoas. Maceió, Alagoas-Brasil. Correo electrónico: nainacalheiros2@gmail.com

${ }^{2}$ Fisioterapeuta, Mestre em gerontologia, Universidade Federal de Pernambuco. Recife-Pernambuco-Brasil. Correo electrónico: laninhabibiano@hotmail.com

${ }^{3}$ Cirurgiã-dentista, Mestranda em Saúde Coletiva, Universidade Federal de Pernambuco. Recife, Pernambuco-Brasil. Correo electrónico: mariliamgoł@hotmail.com

${ }^{4}$ Nutricionista, Mestre em Nutrição Humana, Universidade Federal de Alagoas. Maceió, Alagoas-Brasil. Correo electrónico: taise-gama@hotmail.com

${ }^{5}$ Enfermeiro, Especialista em Saúde da Família, Universidade Estadual de Ciências da Saúde de Alagoas. Maceió, Alagoas - Brasil. Correo electrónico: roberto.firpo@live.com

${ }^{6}$ Psicóloga, Especialista em Saúde do Adulto e do Idoso, Universidade Federal de Alagoas. Maceió, AlagoasBrasil.Correo electrónico: niviamadja@gmail.com

Cómo citar este artículo en edición digital: Sobral, J. P. C. P., Bibiano, A. M. B., Tenório, M. M. G. O., Santos, T. G., Almeida Filho, R. F. E Silva, N. M. S. (2019). La salud del hombre en la perspectiva de la sexualidad: un relato de experiencia. Cultura de los Cuidados (Edición digital), 23(53). Recuperado de http://dx.doi.org/10.14198/cuid.2019.53.27

Correspondencia: Residencial Allegro, Avenida Sebastião Correia da Rocha, 1113. Bloco 04, Apt. 301, Bairro Tabuleiro do Martins. CEP: 57061-410-Maceió (AL), Brasil
\end{abstract}

Correo electrónico de contacto: nainacalheiros2@gmail.com

Recibido: 02/12/2018; Aceptado: 29/01/2019

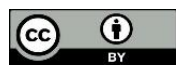

\begin{abstract}
The Men's Health has been a topic widely discussed in recent years, especially after the creation of the National Integral Attention to Men's Health Policy (PNAISH) by the Ministry of Health in 2009. Thus, this article aims at describing the performance of multidisciplinary residents in family health in the implementation and development of a health education group of men in a family
\end{abstract}

health Unit on the theme of sexuality. Was determined to holding weekly meetings with a group of risk of men who meet near their homes, on the sidewalk, under a tree, idle, usually drunk, and making tobacco use and mostly with problems social, psychological and family and who not going for health services. From this, it began the process of conducting health promotion activities with the elected subjects. Realized the importance 
of using innovative methodologies that run through the transfer of information to address issues such as sexuality, which served to strengthen ties between women and health services, as well as to discuss issues that are still said to be taboo.

Keywords: Human health, health education, primary health care, sexuality.

\section{RESUMO}

A Saúde do Homem tem sido assunto amplamente discutido nos últimos anos, em especial após a criação da Política Nacional de Atenção Integral à Saúde do Homem (PNAISH) pelo Ministério da Saúde no ano de 2009. Desta forma, este artigo objetiva relatar a experiência da atuação dos residentes multiprofissionais em saúde da família na implantação e desenvolvimento de um grupo de educação em saúde com homens em uma Unidade de Saúde da Família, sobre a temática da sexualidade. Determinou-se a realização de encontros semanais, com um grupo de homens de risco, que costumavam se encontrar próximo as suas residências, na calçada, embaixo de uma árvore, ociosos, geralmente alcoolizados, e fazendo uso do tabaco e em sua maioria com problemas sociais, psicológicos e familiares e que não procuravam os serviços de saúde. A partir disso, iniciou-se o processo de realização de ações de promoção em saúde com os sujeitos eleitos. Percebeu-se a importância do uso de metodologias inovadoras que transpassem a transferência de informações para abordar questões como a sexualidade, o que serviu para estreitar vínculos entre homens e serviços de saúde, assim como para discutir questões que ainda são ditas como tabus.

Palavras-chave: Saúde do homem, educação em saúde, atenção primária à saúde, sexualidade.

\section{RESUMEN}

La salud del hombre ha sido tema ampliamente discutido en los últimos años, especialmente tras la creación de la Política Nacional de Atención Integral a la Salud del Hombre (PNAISH) por el Ministerio de Salud en el año 2009. De esta forma, este artículo tiene por objeto relatar la experiencia de la salud actuación de los residentes multiprofesionales en salud de la familia en la implantación y desarrollo de un grupo de educación en salud con hombres en una Unidad de Salud de la Familia, sobre la temática de la sexualidad. Se determinó la realización de encuentros semanales, con un grupo de hombres de riesgo, que solían encontrarse cerca de sus residencias, en la calzada, debajo de un árbol, ociosos, generalmente alcohólicos, y haciendo uso del tabaco y en su mayoría con problemas sociales, psicológicos y familiares y que no buscaban los servicios de salud. A partir de eso, se inició el proceso de realización de acciones de promoción en salud con los sujetos elegidos. Se percibió la importancia del uso de metodologías innovadoras que traspasen la transferencia de informaciones para abordar cuestiones como la sexualidad, lo que sirvió para estrechar vínculos entre hombres y servicios de salud, así como para discutir cuestiones que todavía se denominan tabúes.

Palabras clave: Salud del hombre, educación en salud, atención primaria a la salud, sexualidad.

\section{INTRODUÇÃO}

A saúde é um processo socialmente determinado, que se relaciona com o modo de organização e desenvolvimento de uma dada sociedade. Ao extrapolar a esfera biológica, consideram-se as condições de 
vida e trabalho dos indivíduos e coletividades, com diferentes fatores sociais, econômicos, culturais, étnico/raciais, psicológicos e comportamentais influenciando a gênese de diferentes problemas de saúde e seus fatores de risco (Buss e col., 2007). Nesse contexto, a saúde da família como estratégia de reorganização da Atenção Primária à Saúde (APS) surge para consolidar os princípios e diretrizes do Sistema Único de Saúde (SUS) ao concretizar a passagem de uma visão fragmentada para uma visão integral do sujeito, considerando o processo saúde/doença de uma forma mais ampla e complexa (Almeida e col., 2012). Todavia, a organização dos serviços de saúde, especialmente no âmbito da APS, tem resultado em um privilégio no atendimento das necessidades de determinados segmentos sociais como, mulheres, crianças e idosos, em detrimento do público masculino. Além disso, horários de funcionamento coincidem com o horário de trabalho dos sujeitos, e ações de prevenção e autocuidado têm sido pouco direcionadas ao homem e a lógica de atendimento permanece com foco na cura e reabilitação, não havendo garantia da integralidade do cuidado (Silva e col., 2012).

Em contrapartida, há uma construção histórica acerca do que é ser homem, situando-o como um ser viril, invulnerável, que não necessita de cuidados, onde o adoecimento é considerado como sinal de fragilidade. Nessa situação, assume, na maioria das vezes, comportamentos de risco para si e para os que estão a sua volta, não se preocupa consigo e pouco procura pelos serviços de saúde. Esse desenho social contribui para que muitos homens não recebam a devida assistência de saúde que necessitam (Guerreiro, 2012). O homem é ainda considerado do ponto de vista sexual e afetivo. A sexualidade masculina tem sido associada diretamente ao ato sexual, sendo considerada intensa e requerente de satisfação imediata, onde o homem assume o papel de macho sempre pronto para a mulher que está disponível. Ser homem, nesse contexto, também condiz em não ser homossexual. No caso do uso da camisinha, é inconcebível numa relação estável, e as razões que motivam seu uso são apenas a curiosidade e a necessidade de evitar uma gravidez, deixando de lado a utilização para prevenir Infecções Sexualmente Transmissíveis (IST) (Guerreiro, 2012).

Assim, nos moldes da masculinidade ideal estão presentes noções de invulnerabilidade, comportamento de risco e uma sexualidade instintiva e incontrolável, associadas a dificuldades de verbalização das próprias necessidades de saúde e sexuais, pois falar de seus problemas pode significar uma possível demonstração de fraqueza perante os outros (Figueiredo, 2005). Foi diante da necessidade de situar o cuidado à saúde do homem como uma prioridade de atenção e reconhecer este sujeito em sua integralidade, que o Ministério da Saúde instituiu, em 2009, a Política Nacional de Atenção Integral à Saúde do Homem (PNAISH) (Almeida e col., 2012).

Nesse ínterim, a discussão sobre saúde do homem e sexualidade masculina torna-se pertinente, em especial em grupos que trabalham pautados na educação em saúde, onde há um espaço de trocas horizontalizadas com formação de vínculo. É importante ponderar que discutir sexualidade com o homem envolve diferentes questões como, o conhecimento sobre o corpo masculino, a importância da atividade sexual e a vivência do prazer para ambos os sexos, as concepções de gênero e sua importância no cotidiano dos relacionamentos (relações de confiança e fidelidade, por exemplo), bem como a prevenção das doenças (Figueiredo, 2005). Dessa maneira, o objetivo do presente trabalho foi descrever a experiência do desenvolvimento de atividades de educação em saúde sobre sexualidade em um grupo 
de homens de uma comunidade de Maceió, Alagoas.

\section{METODOLOGIA}

Trata-se de um relato de experiência, descritivo, vivenciado em um grupo de educação em saúde para homens, desenvolvido pelos residentes multiprofissionais em saúde da família da Universidade Estadual de Ciências da Saúde de Alagoas (UNCISAL), no período de maio de 2014 a dezembro de 2015. A referida equipe de residentes é composta por sete categorias profissionais, a saber: Educação Física, Enfermagem, Fisioterapia, Nutrição, Odontologia, Psicologia e Terapia Ocupacional que atuam em conjunto na área de abrangência da Estratégia Saúde da Família (ESF), área 72, da Unidade de Saúde da Família (USF) Dr. Hélvio José de Farias Auto, localizada no II Distrito Sanitário de Saúde do Município de Maceió, Alagoas.

Inicialmente, foi realizado o processo de territorialização na área adstrita à USF no intuito de conhecer as especificidades sociais, econômicas, culturais, de morbimortalidade e outros aspectos da área de atuação. Após essa fase, identificou-se a necessidade de construir um grupo voltado para o público masculino, visto que existia um grupo de homens de risco, que costumava se encontrar próximo as suas residências, na calçada, embaixo de uma árvore, ociosos, na maior parte do tempo alcoolizados, e fazendo uso do tabaco e em sua maioria com problemas sociais, psicológicos e familiares e que não procuravam os serviços de saúde.

Determinou-se a realização de encontros semanais com os sujeitos, com o intuito de promover práticas humanizadas de educação em saúde (promoção de saúde e prevenção de doenças). Essa fase se caracterizou pelo estabelecimento de vínculos com os homens por meio de acolhimento e de atividades educativas.
Durante todo o processo de idealização e realização das atividades de educação em saúde, percebeu-se a necessidade de abordar sobre sexualidade, no mês de julho de 2015, em dois dias não consecutivos de atividades, com o objetivo de discutir com esses homens aspectos referentes ao gênero e sexualidade bem como introduzir ações de prevenção as Infecções Sexualmente Transmissível (IST).

\section{RELATO E DISCUSSÃO}

A APS constitui um papel relevante e estratégico para o funcionamento do SUS, pois promove relações contínuas de vínculo com a população, sendo alicerçada em trabalho multi e interdisciplinar que enfoca práticas e ações participativas e democráticas que provoca a autonomia do sujeito e uma corresponsabilização com sua própria saúde (Silva, 2000).

Nesse sentido, a educação em saúde desenvolvida no primeiro dia foi sobre a sexualidade masculina com a dinâmica da Margarida, através da confecção de uma margarida em material de cartolina (Figura 1). No pilar central da flor, havia duas folhas com as palavras: psicológico e biológico, um caule com a palavra cultura e no miolo da flor a palavra sexualidade. Cada pétala da flor tinham as seguintes palavras: prazer, sensualidade, necessidade, orientação sexual, felicidade, emoção, machismo, comunicação, amor, interação, realização, descoberta, carinho, transformação, maturidade, liberdade, reprodução, permissão e IST/AIDS.

A Educação em Saúde é um recurso utilizado por profissionais da saúde para facilitar a comunicação e atuação no cotidiano das pessoas de modo eficaz, respeitando as diferenças. É, portanto, uma ferramenta que leva o empoderamento do sujeito (Silva e col., 2012). 


\section{FIGURA 1: Material educativo utilizado para a discussão sobre a sexualidade masculina com os homens de uma comunidade, Maceió, Alagoas, Brasil, 2015}

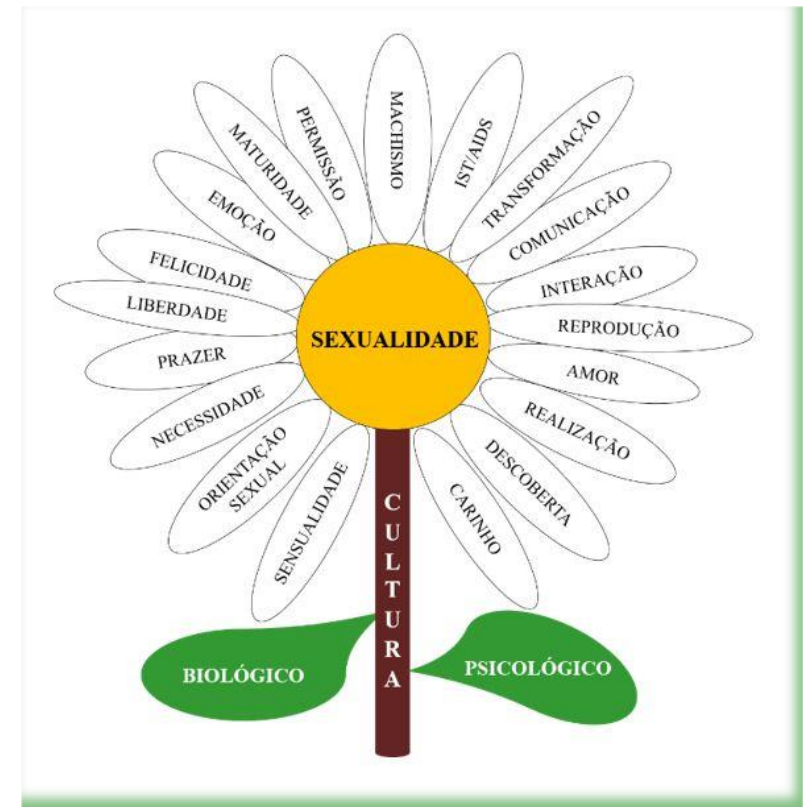

Cada parte descrita da flor foi distribuída para cada homem participante do grupo que foram divididos em duplas, sendo solicitada a reflexão por cinco minutos sobre a relação entre a palavra que recebeu e a sexualidade. Ademais, a dupla colocou no miolo da flor a pétala e verbalizou a reflexão. Ao término da construção da flor, houve a distinção entre sexo e sexualidade, em que o sexo é um dos aspectos da sexualidade e que esta é mais ampla, e se relaciona com a dimensão cultural, psicológica, emocional, fisiológica, e que diz respeito a um conjunto de fatores inerentes a natureza humana.

A sexualidade é por vezes entendida como exercício da atividade sexual, como um funcionamento biológico e psicológico dos sujeitos, porém a compreensão é mais ampla, é tida como produto de diferentes cenários culturais. Considera-se que características entre homens e mulheres são diferentes tanto no aspecto da vida sexual quanto no domínio da reprodução, e são, portanto, uma combinação de fenômenos que ocorrem nos indivíduos como efeito de processos complexos de socialização dos gêneros (Goldenberg e col., 2003).

$\mathrm{Na}$ medida em que os homens colocaram suas reflexões, a equipe de residentes questionava no sentido de mediar a construção coletiva sobre a temática, principalmente quando os comentários vinham enraizados de preconceitos. A discussão teve um desfecho positivo, com debates enriquecedores, nos quais os homens tiveram a oportunidade de se posicionar abertamente. Houve compartilhamento de experiências pessoais, comparações sobre como vivenciamos a sexualidade ao longo da história e também nos diferentes lugares, baseados em histórias pessoais e nos telejornais. Todas as demandas surgidas foram acolhidas, 
respeitadas e debatidas com os participantes.

Pensar na sexualidade masculina é perceber uma exigência socialmente construída, de que o homem seja física e psicologicamente forte, com rejeição pelo autocuidado, adiando ou negando tratamentos preventivos e de promoção e de proteção da saúde. Nesse sentido, torna-se difícil aceitar a doença e reconhecer a relevância que cuidar da saúde e buscar a prevenção é o melhor caminho, evitando com isso o diagnóstico e tratamento tardio (Silva e col., 2012).

O recurso visual, o trabalho em dupla e o vínculo que o grupo já apresentava foram importantes para o desfecho positivo da atividade. A reflexão de gênero se fez presente na discussão, sendo apresentadas as diferenças entre feminino e o masculino, sua construção social e o autocuidado, mas o foco da atividade foi discutir sobre a sexualidade.

"Meninos e meninas crescem sob a crença de que mulher e homem são o que são por natureza". No meio social, a masculinidade diz respeito ao homem que é solitário e isolado no tocante às experiências pessoais, ou, quando muito superficial e prático, deve ser direcionado a atitudes e realização de atividades (Gomes, 2003). Assim, existe uma fragilidade ou o afastamento dos homens das preocupações com o autocuidado e com a busca pelos serviços de saúde, associandose aos valores da cultura masculina como tendências à exposição a riscos, relação da masculinidade à invulnerabilidade e também a própria educação familiar, que traz orientações que o papel social do homem é ser o provedor e protetor (Silva e col., 2012).

A segunda atividade, realizada em outro momento, abordou sobre as Infecções Sexualmente Transmissíveis (IST's) com discussões sobre os principais sinais e sintomas, formas de infecção e tratamento das

IST's mais
(Sífilis/Gonorreia/AIDS/Hepatites), realização de orientações sobre as formas de prevenção. Utilizou-se os seguintes materiais: cartolina, fita adesiva, imagens de órgãos sexuais infectados, imagens de formas de contaminação e prevenção, e alguns símbolos das IST's (laço vermelho e amarelo).

Estudos demonstram que os homens iniciam a atividade sexual precocemente, com várias parceiras, em que são motivados pela atração física e não possuem vínculo afetivo, na busca de provar a masculinidade e autoafirmação de ser homem. A mulher visualiza seu início da vida sexual diferente do homem, pois tende a postergar e esperar o momento e parceiro ideal. Com isso, são influenciados por valores e crenças que defende a superioridade masculina e as desigualdades de gênero (Silva e col., 2010).

Inicialmente, relatou-se o tema da atividade para os participantes e houve a distribuição das imagens individualmente para que associassem às IST's. Na medida em que havia a associação, os homens colavam na cartolina as imagens referentes às informações sobre cada IST's. Caso ocorressem erros, uma prenda teria que ser paga: cantar uma música antiga ou recitar uma poesia. No final, os residentes colocaram o nome das IST's na cartolina e discutiram as formas de contaminação: uso de material perfuro cortante sem distinção, relação sexual desprotegido (oral, anal e vaginal), compartilhamento de alicates de unha, entre outros. Após essa etapa, foi realizada a demonstração do uso correto dos preservativos feminino e masculino com as próteses de borracha. Houve ainda, a demonstração da elasticidade do preservativo masculino, ao testarmos a colocação no braço de um dos presentes, para provar que cabe em todos os tamanhos de pênis, e tentar reduzir o estigma.

Os homens ao se relacionarem sexualmente com várias parceiras, muitas vezes, sem o uso regular do preservativo 
masculino, aumenta sua vulnerabilidade ao acometimento pelo Vírus da Imunodeficiência Humana (HIV) e IST's. Muitos justificam a não adoção da camisinha, pela diminuição do prazer no intercurso sexual, ausência da sensibilidade, desconforto na atividade sexual, irritação vaginal, risco de rompimento, entre outros. Por isso, as ações de educação em saúde são relevantes por levar orientações na tentativa de reduzir comportamentos de risco (Silva e col., 2010).

A atividade foi dinâmica, com diversificadas informações visando à prevenção das IST's. O grupo demonstrou conhecimento sobre a temática, o que reforça a necessidade de sensibilizar para o uso do preservativo e outras formas de prevenção, e não meramente um repasse de informação sobre as doenças. Por fim, foram distribuídos preservativos masculinos, femininos e gel lubrificante, e também orientamos sobre a importância de tomar as três doses da vacina contra hepatite do tipo B.

Vale ressaltar que a doença mais conhecida por esse grupo foi a AIDS, pois entre as IST's, possui maior poder de disseminação e letalidade (Rebello e col., 2011). Diante disso, faz-se necessário provocar espaços de discussões voltadas para os sentidos atribuídos à sexualidade masculina, que não se resumam apenas na prevenção do câncer de próstata, mas também de outras doenças e saúde sexual, que enfoque o autocuidado e a busca pelo serviço de saúde (Gomes, 2003).

\section{CONSIDERAÇÕES FINAIS}

Este estudo traz uma discussão ainda acanhada, mas que vem tomando força e mostra através de suas atividades que é necessário permitir que os homens possam expressar suas dúvidas, ansiedades e fragilidades, principalmente diante de um conceito de masculinidade que vem se alterando, para que se sintam mais acolhidos e possam procurar ajuda às suas questões de saúde.

No que se refere às ações educativas, percebe-se que elas ganham espaço junto a medidas de prevenção de IST/AIDS, tornando-se um meio de debate importante para tirar dúvidas e estreitar vínculos com os profissionais de saúde, diferente dos métodos tradicionais de campanhas. Essas ações passam a ser uma opção e poderão ser mais exitosas na medida em que, por sua metodologia, consigam transpor o limite da transmissão de informações para as discussões que problematizam as medidas preventivas bem como permeadas por questões que circundam as relações de gênero.

Percebe-se também a necessidade de se aprofundar mais as discussões sobre esses temas, visto que ainda têm sido feitas de forma superficial e pontual e de modo a não a reduzir à instância do se evitar a doença, mas ressignificá-la como caminho de promoção de interações afetivo-sexuais mais saudáveis.

\section{REFERÊNCIAS}

- Almeida, L. P., Silva, S. O. \& Silva, M. M. (2012). Grupo de educação em saúde para homens: um relato de experiência da enfermagem. Revista de Enfermagem, 8(8), 227-234.

- Buss, P. M. \& Pellegrini Filho, A. (2007). A Saúde e seus Determinantes Sociais. PHYSIS: Rev. Saúde Coletiva, 17(1), 77-93.

- Figueiredo, W. (2005). Assistência à saúde dos homens: um desafio para os serviços de atenção primária. Ciência \& Saúde Coletiva, 10(1), 105-109.

- Goldenberg, P., Marsiglia, R. M. G. \& Gomes, M. H. A (Org.). (2003) O Clássico e o Novo: tendências, objetos e abordagens em ciências sociais e saúde. Rio de Janeiro: Editora Fiocruz.

- Gomes, R. (2003). Sexualidade masculina e saúde do homem: proposta para uma discussão. Ciência \& Saúde Coletiva, 8(3), 825-829.

- -Guerreiro, I. C. Z. (2012). Masculinidades e prevenção do HIV. Bol. Inst. Saúde (impr.), 14(1), 
1809-7529.

- Rebello, L. E. F. S., Gomes, R. \& Souza, A. C. B. (2011). Men and AIDS prevention: analysis onknowledge production within the field of healthcare. Interface-Comunic. Saude Educ., 15(36), 67-78.

- Silva, S. G. (2000). Masculinidade na história: a construção cultural da diferença entre os sexos. Psicol. cienc. prof., 20(3), 8-15.

- Silva, K. L., Dias, F. L. A., Maia, C. C., Pereira, D. C. R., Vieira, N. F. C. \& Pinheiro, P. N. C. (2010). A influência das crenças e valores culturais no comportamento sexual dos adolescentes do sexo masculino. Rev. enferm. UERJ, 18(2), 247-252.

- Silva, P. A. S., Furtado, M. S., Guilhon, A. B., Souza, N. V. D. O. \& David, H. M. S. L. (2012). A saúde do homem na visão dos enfermeiros de uma Unidade Básica de Saúde. Esc Anna Nery (impr.), 16(3), 561568 . 